\begin{tabular}{|c|c|c|c|}
\hline Pan & Portuguese of Angola & $\mathrm{ScGl}$ & Scottish Gaelic \\
\hline Pbr & Portuguese of Brasil & Scp & Spanish Creole \\
\hline \multirow[t]{2}{*}{ Pcp } & Portuguese Creole of & & Palenquero \\
\hline & Príncipe & Scr & Serbo-Croatian \\
\hline Pct & $\begin{array}{l}\text { Portuguese Creole of } \\
\text { São Tomé }\end{array}$ & Sdr & $\begin{array}{l}\text { Spanish of the } \\
\text { Dominican Republic }\end{array}$ \\
\hline Pol & Polish & Slk & Slovak \\
\hline Por & Portuguese & Slv & Slovenian \\
\hline Pro & Provençal & Spn & Spanish \\
\hline Rmns & Rhaeto-Romansh & Srd & Sardininan \\
\hline Rum & Rumanian & Swd & Swedish \\
\hline Rus & Russian & Trk & Turkish \\
\hline San & Sango & Ukr & Ukrainian \\
\hline Sar & Sara & Wls & Welsh \\
\hline
\end{tabular}

\title{
Abbreviations of authors and texts
}

(Abbreviations of texts are given in italics)

\begin{tabular}{|c|c|c|c|}
\hline Aesch. & Aeschylus & $K B o$ & Keilschrifttexte aus \\
\hline Bacch. & Bacchides & & Boğazköy [Texts in \\
\hline Cas. & Casina & & cuneiform script from \\
\hline Cat. & Orations against & & Boğazköy] \\
\hline & Catiline & Le. & The Gospel according \\
\hline Curc. & Curculio & & to Luke \\
\hline Cor. & $\begin{array}{l}\text { Epistle to the } \\
\text { Corinthians }\end{array}$ & L. $L$. & $\begin{array}{l}\text { De lingua latina [On } \\
\text { the Latin language] }\end{array}$ \\
\hline Cycl. & Cyclops & Lucr. & Lucretius \\
\hline$D B$ & $\begin{array}{l}\text { Darius' Inscription at } \\
\text { Behistan }\end{array}$ & $\begin{array}{l}\text { Mart. } \\
\text { Mc. }\end{array}$ & $\begin{array}{l}\text { Martial } \\
\text { The Gospel according }\end{array}$ \\
\hline Gal. & $\begin{array}{l}\text { Epistle to the } \\
\text { Galatians }\end{array}$ & Mil. & $\begin{array}{l}\text { to Mark } \\
\text { Miles gloriosus [The }\end{array}$ \\
\hline Eurip. & Euripides & & boastful soldier] \\
\hline Hel. & Heliand & Od. & Odyssey \\
\hline Hom. & Homer & Otfr. & Otfried \\
\hline Il. & Iliad & Nibel. & Nibelungenlied \\
\hline Job. & $\begin{array}{l}\text { The Gospel according } \\
\text { to John }\end{array}$ & $\begin{array}{l}\text { Plaut. } \\
\text { Prom. }\end{array}$ & $\begin{array}{l}\text { Plautus } \\
\text { Prometheus vinctus }\end{array}$ \\
\hline
\end{tabular}




$\begin{array}{llll}\text { Pseud. } & \text { Pseudolus } & \text { Tat. } & \text { Tatian } \\
\text { RV } & \text { Hymns of the } & \text { Tim. } & \text { Epistle to Timothy } \\
& \text { RigVeda } & \text { Truc. } & \text { Truculentus } \\
\text { XII Tab. } & \text { Leges duodecim } & \text { Varr. } & \text { Varro } \\
& \text { tabularum [The laws } & \text { Vid. } & \text { Vidēvdād } \\
& \text { of the twelve tables] } & Y . & \text { Yašt } \\
\text { Tab.Ig. } & \text { Tabulae Iguvinae } & & \\
& \text { [Tables from } & & \\
& \text { Gubbio] } & & \end{array}$


